$5^{\text {th }}$ US Combustion Meeting

Organized by the Western States Section of the Combustion Institute and Hosted by the University of California at San Diego

March 25-28, 2007

\title{
Application of Model Fuels to Engine Simulation
}

\author{
K. V. Puduppakkam, J. M. Deur, , D. Hodgson, E. Meeks, S. F. Miller, \\ M. V. Petrova, , C. Wang, C. K. Westbrook \\ Reaction Design
}

\author{
A. M. Dean \\ Colorado School of Mines \\ W. H. Green \\ Massachusetts Institute of Technology \\ M. Koshi \\ University of Tokyo \\ U. Maas \\ Universität Karlsruhe
}

\begin{abstract}
To address the growing need for detailed chemistry in engine simulations, Reaction Design has founded the Model Fuels Consortium, together with key members from the automotive and fuels industry. The Consortium is implementing a 3-year roadmap of industry-defined goals for engine-combustion simulation. This involves developing a comprehensive set of model-validation documentation for targeted experimental data, including both fundamental experiments and engine data. In addition, we are expanding the database of mechanisms for fuel components and are developing software tools that extend analysis capability, including automated reduction of reaction mechanisms for targeted simulation conditions. Selected results are presented and discussed.
\end{abstract}

\section{INTRODUCTION}

To simulate the performance of fuel combustion adequately in a diesel or HCCI engine, the detailed chemical kinetics of the combustion process must be considered. Chemical kinetics are critical to simulating ignition performance and controllability, as well as engine emissions. Real fuels, such as diesel, gasoline or jet fuels, consist of several hundreds of chemical components, which makes direct simulation intractable. However, research has shown that selected mixtures of a much smaller number of model fuel compounds can adequately represent many fuel/engine characteristics. The advantage of the model-fuel approach is that the model fuel consists of well defined chemical species, for which detailed oxidation and pyrolysis chemistry models can be developed. Model fuel mixtures can be selected to match characteristics of a corresponding real fuel, including heat-release rates, auto-ignition timing, $\mathrm{NO}_{\mathrm{x}}$ emission, and sooting propensity.

The use of surrogate or model fuels requires detailed chemistry. Engine simulations require chemistry models that describe ignition phenomena, heat-release, and pollutant formation under engine-combustion conditions. Currently, many important model-fuel components are not well characterized and systematic validation studies under engine-relevant conditions are 
scarce. Furthermore, detailed mechanisms are often too large to be handled in realistic engine-design simulations. There is therefore a growing and critical need for a broadly populated model-fuel reaction database, automated mechanism-reduction tools, comprehensive validation comparisons of detailed chemistry models to fundamental experiments and engine data, and software tools that facilitate the use of chemistry data in engineering flow simulations.

The Model Fuels Consortium (MFC) was founded to address this need. Current members of the consortium include: Chevron, ConocoPhillips, The Dow Chemical Company, L'Institut français du pétrole (IFP), Honda R\&D Company, Mazda Motor Corporation, Mitsubishi Motors, Nissan Motor Company, PSA Peugeot Citroën, and Toyota Motor Corporation. The objective is to advance the ability of members to use and manipulate detailed chemistry information for model fuels in engine and fuels-combustion simulation. Tasks that have been performed in the first year include the following:

1. Generation of detailed chemistry models for new model-fuel components that allow more accurate descriptions of real fuels

2. Documentation of a comprehensive study of chemistry-model performance against a wide range of experimental data, for an initial set of model fuels

3. Implementation of automated mechanism-reduction methods that can be applied to 0-D transient and 1-D simulations over a range of conditions

4. Development of an Octane Number Calculator, which predicts the Research and Motor octane numbers using detailed fuel-combustion models

5. Development of a graphical reaction-path analyzer that provides interactive analysis of reaction contributions to species production

6. Establishment of a database that facilitates comparison of chemistry models originating from different authors, based on definitive species identification.

Here we highlight results from the mechanism validation study and the automation of mechanism-reduction.

\section{RESULTS AND DISCUSSION}

\section{INITIAL FUEL CHEMISTRY MODELS}

The initial set of fuel components studied by the consortium include n-heptane, iso-octane, toluene, 1-pentene, methyl cyclohexane, and their mixtures. The initial mechanisms used in the modeling are based on publications from the Lawrence Livermore National Laboratories Combustion Chemistry group and their collaborators. ${ }^{1-6}$ The reaction mechanism that contains all 5 components includes 5835 reactions and 1328 species. The goal was to provide an objective evaluation of the ability of the detailed chemistry models to describe fundamental phenomena that is critical to internal-engine combustion behavior. The components studied are the basic building blocks for constructing model-fuel mixtures that are used to represent diesel and gasoline fuels in simulation.

\section{VALIDATION OF DETAILED CHEMISTRY MODELS}

Model comparisons have been performed against experimental data that includes measurements from shock-tubes, rapid compression machines (RCMs), flow reactors, jetstirred reactors, both premixed and non-premixed flames, and laboratory engines. Experimental data have been collected from the literature and from engine experiments 
performed for specific use in model evaluation for the MFC. For each set of experiments, a model of the experiment was developed using CHEMKIN simulation software. ${ }^{7}$ Where nonidealities in the experimental system may affect the simulation agreement with data, an evaluation of the effects of the model deficiencies were included in the consideration of the comparison findings. The results of the study are recommendations for both reactionmechanism improvements and extensions required of the simulation software for improvement of the experimental representation.

Over 100 CHEMKIN projects were assembled for the validation study, with each project representing many computational runs that vary one or more experimental parameters, such as temperature, pressure, and equivalence ratio. The CHEMKIN models consisted of an internal combustion engine cycle model, closed-homogeneous reactors under a variety of volume or pressure constraints, perfectly stirred reactors, 1-dimensional premixed flames, and opposed-flow non-premixed and partially premixed flames. Each project has been saved and archived, along with the corresponding experimental data, such that the comparison can be readily repeated as the mechanisms are improved over the course of the Consortium project.

Figure 1 shows the wide range of experimental data points considered, for example, for nheptane ignition-delay. For each temperature value, a range of pressures, equivalence ratios, and degrees of dilution were used in the various experiments, providing the vertical spread in the points shown in Figure 1.

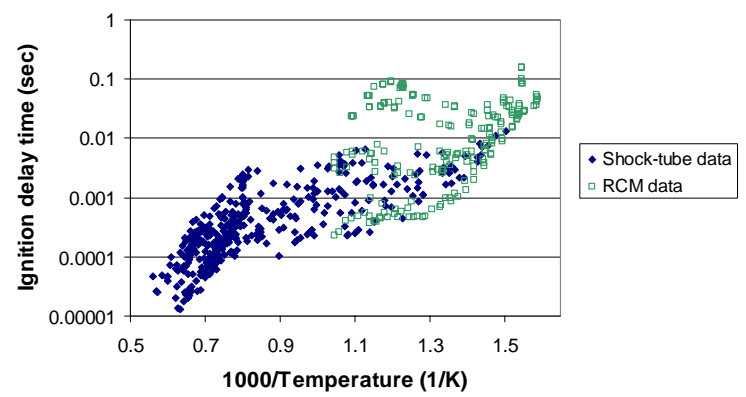

Figure 1 Range of ignition-delay data considered for $n$-heptane, including variations in pressure and mixture stoichiometry.

A representative set of comparisons for the model vs. experiment is shown in Figure 2, while Figure 3 indicates where the points in Figure 2 reside in the map of experimental points shown in Figure 1. As previously reported ${ }^{5}$, the detailed n-heptane reaction mechanism does well in capturing the negative temperature-coefficient region for the ignition delay vs. 1/T curve, where the ignition-delay is actually increased as the temperature is increased for moderate temperatures. 


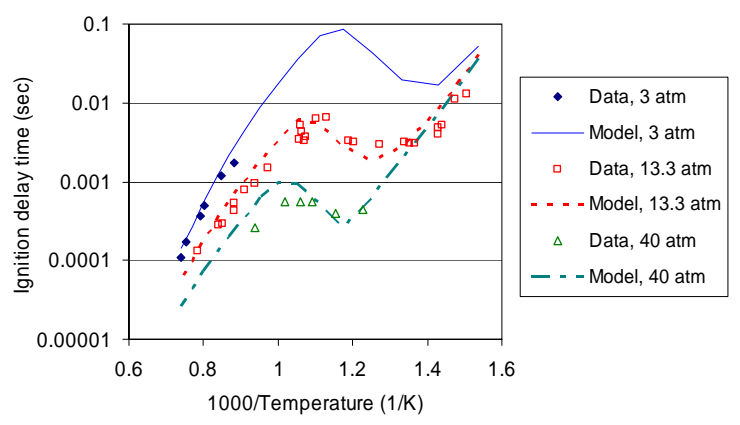

Figure 2 Model comparison for n-heptane with the shock-tube data of Ciezki et al. ${ }^{8}$

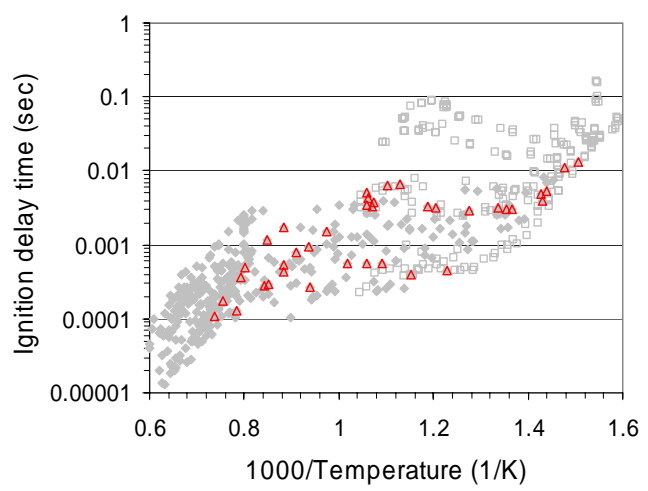

Figure 3 Location of Ciezki, et al. data on map of all experimental points for $\mathbf{n}$-heptane.

An example of comparisons for fuel mixtures is shown in Figure 4, where a model of the flow-reactor experiment of Callahan, et al. ${ }^{9}$ for a primary reference fuel (iso-octane/nheptane) mixture (62\% iso-octane) is compared with species-profile measurements.

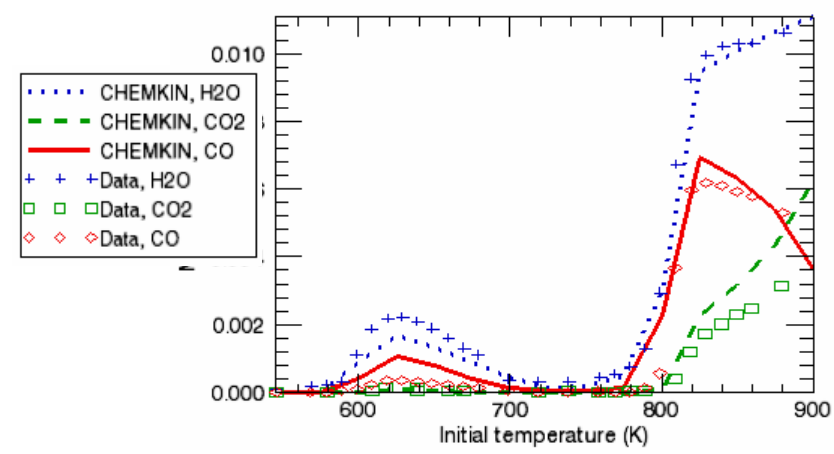

Figure 4 Comparison of model predictions vs. data for flow-reactor conditions of Callahan, et al. ${ }^{9}$

In many cases, areas of non-agreement were also revealed, particularly in low-temperature kinetics for some of the less well studied fuels. In addition, significant discrepancies in flame-speed predictions were identified under certain conditions. For these cases, sensitivity and uncertainty analysis have been applied to determine the sensitivity of the model comparison to different model-input parameters. Improvements of the models are underway, which will become part of an ongoing and documented validation-improvement cycle. 
Here we emphasize the systematic approach to providing a baseline set of simulation comparisons, which can then be easily revisited with updates to either the chemical-model or the simulation representation of the experiment. From the baseline comparison, we can also determine reasonable uncertainties in important model-predicted variables that are important to engine simulation today, such as ignition-delay time and laminar flame speed.

\section{AUTOMATION OF MECHANISM REDUCTION}

In addition to generating and testing detailed chemistry models for model-fuel components, we have also developed a prototype of an automated facility to provide skeletal versions of the detailed or "master" mechanisms. The goal of this project was to apply mechanismreduction strategies for a relatively wide range of conditions and to provide a mechanism that is significantly smaller in both the number of species and the number of reactions. In this context, "skeletal" means that the mechanism still contains elementary reactions and chemically defined molecules, but reactions and species that do not contribute to the behavior of interest have been removed.

After evaluating several optional methods, the Directed Relational Graph (DRG) method reported by $\mathrm{Lu}$ and $\mathrm{Law}^{10}$ was selected. This approach has the advantage of requiring very little end-user knowledge about the chemistry model and provided reasonably accurate results over a range of conditions. This method was implemented with the CHEMKIN simulation software, such that it can be applied to a parameter study (where one or more parameters are systematically varied) involving any reactor model.

Figure 5 demonstrates the result for n-heptane ignition-delay. Results are shown for an equivalence ratio of 2 and pressure of $40 \mathrm{~atm}$. Figure 5 compares results for a skeletal mechanism (256 species and 1369 reactions) against the same simulations using the full, master mechanism for n-heptane, which contains 561 species and 2539 reactions. The reduction was run to cover the range of equivalence ratios from 0.1 to 2.0, for temperatures between $600 \mathrm{~K}$ and $1800 \mathrm{~K}$, and for pressures from $0.5 \mathrm{~atm}$ to $60 \mathrm{~atm}$, represented by 384 CHEMKIN simulations. For this level of reduction, the error is almost imperceptible between the reduced and original model. Similar agreement is found for equivalence ratio of 0.1 and pressures as low as $0.5 \mathrm{~atm}$.

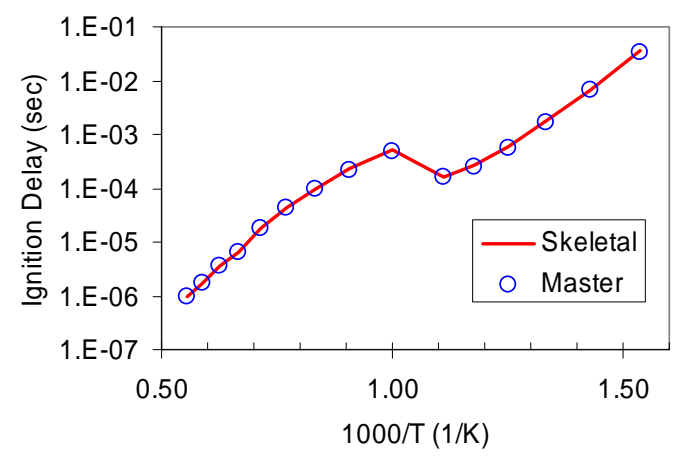

Figure 5 Comparison of ignition-delay predictions for a skeletal mechanism vs. the original, master mechanism for $n$-heptane.

Similar results are shown in Figure 6 for a set of flame-speed calculations, where the skeletal mechanism for this case contained 121 species and 538 reactions. Since flame speed 
calculations involve only high-temperature reactions, a smaller mechanism can be obtained while still achieving accurate results, compared to ignition-delay calculations that may include both low and high-temperature kinetics. Results are shown in Figure 6 for flamespeed calculations at atmospheric pressure and for an initial temperature varying from 300 to $600 \mathrm{~K}$.

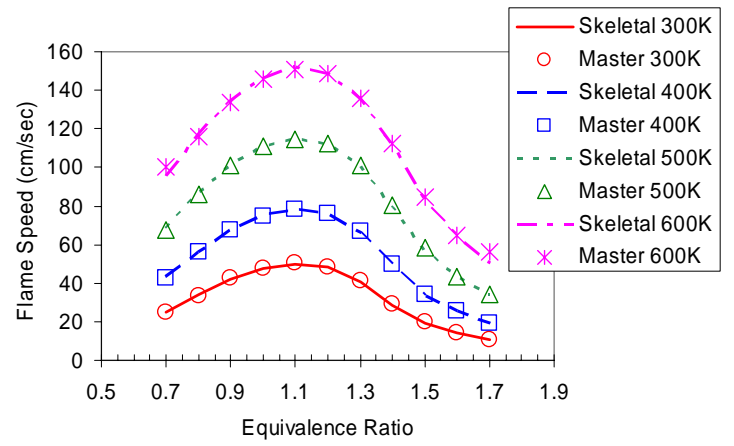

Figure 6 Comparison of flame-speed calculations for skeletal vs. full mechanism for nheptane.

These results demonstrate the effectiveness of the DRG approach, when applied to a parameter-study containing a wide range of conditions. Further reduction can be achieved for smaller target ranges or with the acceptance of larger error. The effect of the size reduction shown, however, can provide a dramatic decrease in simulation time, as illustrated in Figure 7. These results are for simulations on a computer with a $2.8 \mathrm{GHz}$ CPU and 8GB RAM.

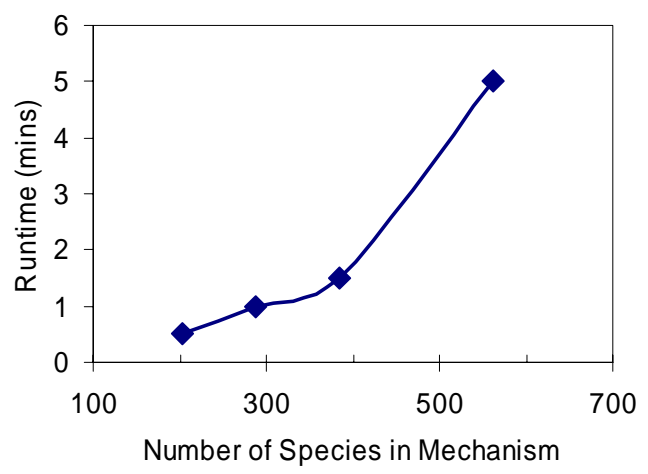

Figure 7. Dependence of run-time for ignition-delay calculations on the number of species in the reaction mechanism, for $n$-heptane.

Further reduction while maintaining the mechanism flexibility and accuracy will likely require different mechanism-reduction techniques, such as species lumping, use of partial equilibrium assumptions, ${ }^{11}$ or identification of time-scale manifolds within the kinetic system. ${ }^{12}$ The DRG method, however, provides an important first step in reducing very large reaction mechanisms to a manageable size prior to further reduction. The overlay method that we have implemented allows application of this automated-reduction methodology to a wide range of conditions where several parameters are varied. 


\section{CONCLUSION}

The results presented here provide representative results from the work being performed under the Model Fuels Consortium, where a systematic approach is being taken to expand the set of available, verified data for use in fuels simulation relevant to engine design.

The results of comprehensive validation studies show that predictive capabilities are quite good for some of the more well studied fuels, such as n-heptane, for a very wide range of conditions and phenomena of interest. At the same time, specific improvements needed have been identified for other fuel components.

Once a set of detailed mechanisms have been verified for a wide range of conditions, these mechanisms can be reduced to target a more limited application in engineering design. Reduction is typically necessary due to the (often prohibitive) requirement of computational resources for the direct use of the full mechanisms for larger hydrocarbons. For this purpose, automated reduction is necessary, such that end-users of the data can easily generate a targeted mechanism for a new set of conditions, based on the original (verified) mechanism. In this work we have demonstrated the implementation of DRG method which can be applied to a specified range of conditions, using CHEMKIN-based simulations.

\section{REFERENCES}

1. H. J. Curran, P. Gaffuri, W. J. Pitz, and C. K. Westbrook, "A Comprehensive Modeling Study of Iso-Octane Oxidation," Combustion and Flame, 129: 253-280, 2002.

2. M. Ribaucour, R. Minetti, L. R. Sochet, H. J. Curran, W. J. Pitz, and C. K. Westbrook, "Ignition of isomers of pentane: an experimental and kinetic modeling study," Proceedings of the Combustion Institute, 28: 1671-1678, 2000.

3. C. K. Westbrook, "Chemical kinetics of hydrocarbon ignition in practical combustion systems," Proceedings of the Combustion Institute, 28: 1563-1577, 2000.

4. W. J. Pitz, R. Seiser, J. W. Bozzelli, I. Da Costa, R. Fournet, F. Billaud, F. Battin-Leclerc, K. Seshadri, and C. K. Westbrook, "Chemical Kinetic Characterization of Combustion of Toluene," in 2nd Joint Meeting of the U.S. Section of the Combustion Institute. Oakland, CA: U.S. Dept. of Energy, 2001.

5. H. J. Curran, P. Gaffuri, W. J. Pitz, and C. K. Westbrook, "A Comprehensive Modeling Study of n-Heptane Oxidation," Combustion and Flame, 114: 149-177, 1998.

6. C. V. Naik, W. J. Pitz, M. Sjoberg, J. E. Dec, J. Orme, H. J. Curran, J. M. Simmie, and C. K. Westbrook, "Detailed Chemical Kinetic Modeling of Surrogate Fuels for Gasoline and Application to an HCCI Engine " Fall Powertrain and Fluid Systems Conference \& Exhibition, Society of Automotive Engineers, San Antonio, Texas, 2005.

7. CHEMKIN Release 4.1, Reaction Design, San Diego, CA, 2006.

8. H. K. Ciezki and G. Adomeit, "Shock-Tube investigation of self-ignition of $n$-heptane-air mixtures under engine relevant conditions," Combustion and Flame, 93: 421-433, 1993.

9. C. V. Callahan, T. J. Held, F. L. Dryer, R. Minetti, M. Ribaucour, and L. R. Sochet, "Experimental data and kinetic modeling of primary reference fuel mixtures," 26th Symposium (International) on Combustion, 1996.

10. T. Lu and C. K. Law, "A directed relation graph method for mechanism reduction," Proceedings of the Combustion Institute, 30: 1333-1341, 2005.

11. J.-Y. Chen, "A general procedure for constructing reduced reaction mechanisms with given independent relations," Sandia National Laboratories 87-8782, 1987. 
12. U. A. Maas and S. B. Pope, "Simplifying chemical kinetics: intrinsic low-dimensional manifolds in composition space," Combustion and Flame, 88: 239-264, 1992. 